\title{
O IMPACTO DAS FAKE NEWS NA VACINAÇÃO E NOS SURTOS DE DOENÇAS ERRADICADAS
}

\section{THE IMPACT OF FAKE NEWS ON VACCINATION AND OUTBREAKS OF ERADIC DISEASES}

\author{
Davi Azevedo Ferreira ${ }^{1}$ \\ Alison Pontes da Silva ${ }^{2}$ \\ Camila de Albuquerque Montenegro ${ }^{3}$
}

RESUMO: Introdução: As fakes News (FN) alcançaram um amplo destaque nas notícias, influenciando intensamente a vida, transformando modos de pensar. Um dos exemplos de mudança é acerca das atitudes perante a vacinação, verificado pelo aumento ou retorno das doenças, algumas já erradicadas em nosso país. Objetivo: Constatar o impacto das FN na vacinação e os surtos de doenças, destacando as erradicadas na população. Método: Foi feita uma revisão sistemática da literatura nas bibliotecas: scielo, pubMed e lilacs, de artigos publicados entre os anos de 2011 a 2020, nos idiomas português e inglês, usando os descritores: doenças, surto de doenças (SD), vacinação e mídias sociais (MS). Após combinação dos descritores, foram incluídos ou excluídos do quantitativo final a partir da leitura dos títulos. Posteriormente, à análise dos resumos que contemplavam a relação das FN com vacinação, diminuição da vacinação na população, surtos de doenças e vacinação, compuseram a amostra final. Resultados e discussão: Dos 514 resultados, foi selecionado 50 estudos, e com a análise dos resumos excluídos 25 , sendo selecionados para o estudo, 25 artigos. A maior quantidade de artigos encontrados foi no Pubmed, e com a temática vacinação e doenças erradicadas (8). Evidenciou- se grande variedade das temáticas abordadas pelos descritores escolhidos, como artigos que contemplavam a vacinação, bem como suas doenças erradicadas e como as FN podem interferir para prejudicar a vacinação. Conclusão: Apesar das vacinas protegerem boa parte da sociedade, é preocupante a crescente população que está comprometendo a cobertura vacinal e a saúde coletiva ao depositar incredibilidade em FN.

\footnotetext{
${ }^{1}$ Graduando do curso de Farmácia da Universidade Federal de Campina Grande, Campus Cuité. daviazevedoferreira@hotmail.com.

${ }^{2}$ Graduando do curso de Farmácia da Universidade Federal de Campina Grande, Campus Cuité. alisonpds2@gmail.com.

${ }^{3}$ Professora doutora do Centro de Educação e Saúde na Universidade Federal de Campina Grande, Campus Cuité, área de atuação: Assistência farmacêutica. camontenegro2502@gmail.com.
} 
Palavras chave: Antivacinação, Comunicação, Doenças Contagiosas, Mídias Sociais, Vacinas.

ABSTRACT: Introduction: Fakes News (FN) reached a wide prominence in the news, influencing life intensely, transforming ways of thinking. One example of change is about attitudes towards vaccination, verified by the increase or return of diseases, some of which have already been eradicated in our country. Objective: To verify the impact of NFs on vaccination and disease outbreaks, highlighting those eradicated in the population. Method: A systematic literature review was carried out in the libraries: scielo, pubMed and lilacs, of articles published between the years 2011 to 2020, in Portuguese and English, using the descriptors: diseases, disease outbreak (DS), vaccination and social media (MS). After combining the descriptors, they were included or excluded in the final amount from the reading of the titles. Subsequently, the analysis of the abstracts that contemplated the relationship between $F N$ and vaccination, decreased vaccination in the population, disease outbreaks and vaccination, comprised the final sample. Results and discussion: Of the 514 results, 50 studies were selected, and with the analysis of the excluded abstracts 25, 25 articles were selected for the study. The largest number of articles found was in Pubmed, and with the theme of vaccination and eradicated diseases (8). A great variety of the themes addressed by the chosen descriptors was evidenced, such as articles that contemplated vaccination, as well as their eradicated diseases and how NFs can interfere to harm vaccination. Conclusion: Although vaccines protect a good part of society, it is worrying the growing population that is compromising vaccination coverage and public health by depositing incredulity in FN.

Keywords: Anti-vaccination, Communication, Contagious Diseases, Social Media, Vaccines. 


\section{INTRODUÇÃO}

O termo fake news (FN) alcançou um grande destaque contemporâneo, mesmo tendo sido citado e identificado, pela primeira vez, em 1925, por McKernon. Com o crescimento da internet, inicia-se, mundialmente, uma mudança em meio as comunicações, modificando, assim, o modo como as notícias chegam às pessoas e a sua veracidade, levando consequências à vida da população (WANG et al., 2019).

Ao longo da história da ciência, surge a vacinação como importante contribuidora para o desenvolvimento humano, animal e instalação da saúde, especialmente nos países em desenvolvimento. A mortalidade por varíola e sarampo foi enorme no período pré-vacinal, com até metade da população morta durante epidemias e sarampo foi apenas um pouco menos letal em populações suscetíveis. Com a vacinação, esses dados foram diminuindo até a erradicação dessas doenças (GREENWOOD, 2014).

As vacinas representam um dos principais aspectos da prevenção pediátrica. Entretanto, os pais estão, cada vez mais, questionando sobre a segurança e a necessidade de vacinas, e, como resultado, taxas de vacinação têm níveis perigosamente baixos em certas comunidades. Atrelado a isso, vem a disseminação de notícias falsas, que diminuem, ainda mais, a cobertura vacinal (MCCLURE; CATALDI; O'LEARY, 2017).

Desinformação e falsidade das notícias no âmbito da saúde, espalhadas pelas mídias sociais, constituem uma ameaça em potencial à saúde pública e coletiva. $40 \%$ dos links compartilhados com mais frequência mostram notícias falsas. $\mathrm{O}$ conteúdo mais falicioso diz respeito às vacinas e à vacinação. $\mathrm{A}$ análise das principais notícias compartilhadas das mídias sociais pode contribuir para a identificação das informações médicas falsas mais relevantes, desmembrando a sociedade, causando danos severos, como problemas graves de saúde, que podem levar ao óbito (WASZAK; KASPRZYCKA-WASZAK; KUBANEK, 2018). 
As notícias falsas são uma temática estudada amplamente no meio da comunicação, entretanto, pouco relacionada com a saúde das populações. O debate desse estudo é de suma importância no tocante à vacinação infantil, pois nessa faixa etária a imunidade está em fase de desenvolvimento e a criança sujeita às infecções oportunistas (LIRIA, 2017). Diante disso, este trabalho objetivou observar o impacto das FN na vacinação e os surtos de doenças, com ênfase naquelas já erradicadas na população.

\section{METODOLOGIA}

Para isso, produziu-se esta revisão sistemática feita a partir de uma reunião/integração das hipóteses apresentadas de estudos primários que continham objetivos, materiais e métodos bem explicados, de forma clara e reprodutível (LOPES; FRACOLLI, 2008). Inicialmente, pesquisou-se os artigos nas seguintes bibliotecas virtuais: scielo, pubMed e lilacs, publicados entre os anos de 2011 a 2020, para um alcance maior de estudos, nos idiomas português e inglês. Foram usados quatro descritores para a análise: Doenças, surto de doenças (SD), vacinação e mídias sociais (MS), inclusive em combinações, sendo traduzidos para inglês na hora das buscas nas bases, para abranger uma quantidade significativa de artigos.

Após combinação com os descritores, foram submetidos à leitura dos títulos. Procedeu- se com a análise dos resumos, por meio da qual a amostra passou a ser constituída por um quantitativo menor que o inicial, pois contemplavam todas as informações necessárias, sendo elas a relação das FN com a vacinação, bem como com a diminuição da vacinação na população, além de possuírem as temáticas $F N$, surtos de doenças e vacinação em seus artigos. Os artigos que não apresentavam essas características foram excluídos da análise (figura 1). 
Figura 1- Processo de busca de artigos.

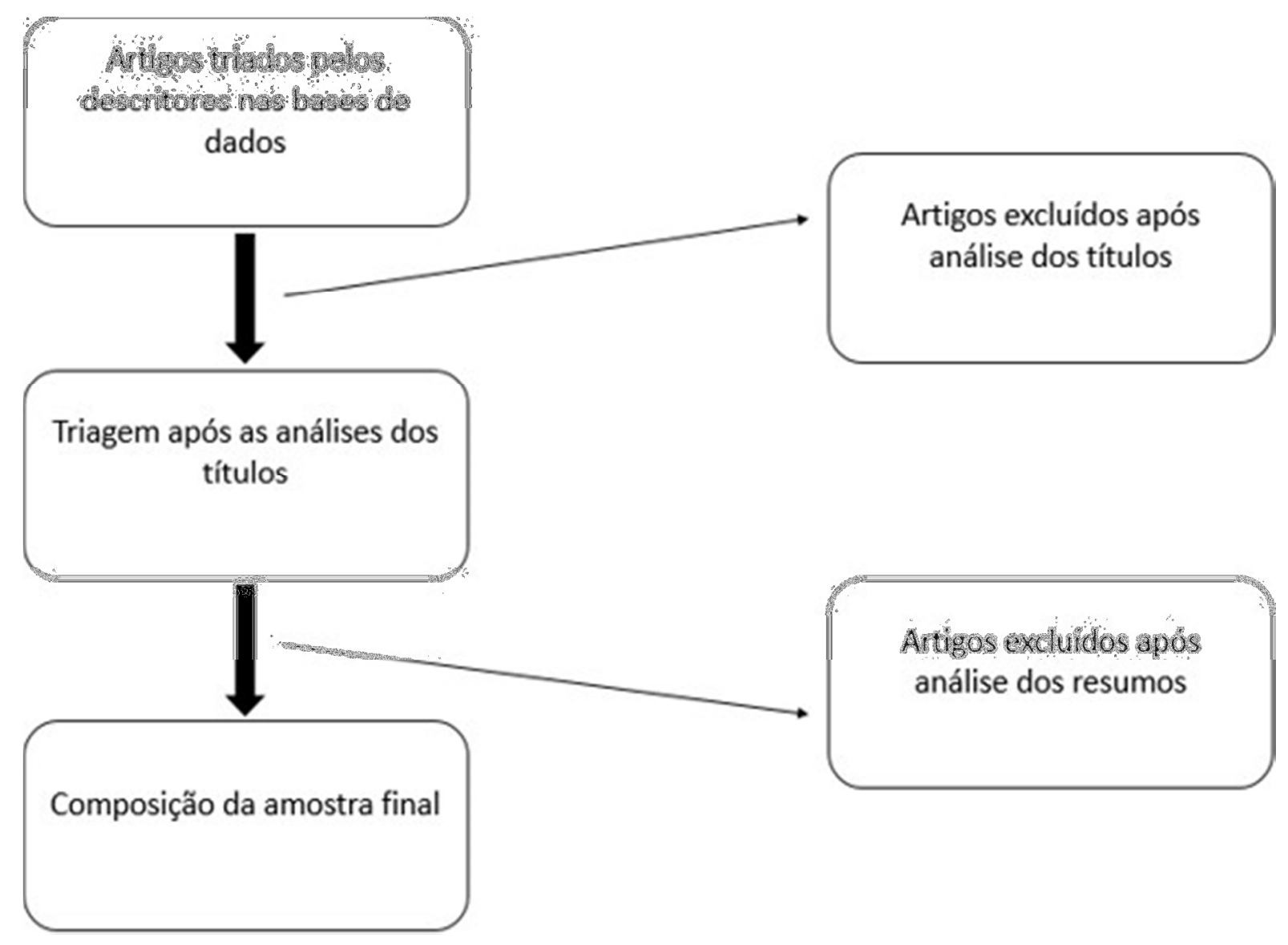

Fonte. Dados da pesquisa, 2020.

\section{RESULTADOS E DISCUSSÃO}

A partir dos estudos selecionados, pode-se observar que a maior quantidade de artigos foi no Pubmed, e com a combinação de descritores: vacinação e SD (quadro 1). 
Quadro 1 - Quantidade de artigos por base de dados e seus descritores.

\begin{tabular}{|c|c|c|c|c|c|}
\hline & $\begin{array}{c}\text { MS + } \\
\text { Vacinação }\end{array}$ & MS+SD & $\begin{array}{c}\text { Vacinação } \\
+ \text { SD }\end{array}$ & $\begin{array}{c}\text { MS } \\
\text { +Doenças }\end{array}$ & Total \\
\hline LILACS & 1 & 0 & 2 & 0 & 3 \\
\hline SciELO & 0 & 0 & 2 & 0 & 2 \\
\hline PubMed & 11 & 0 & 470 & 27 & 508 \\
\hline $\begin{array}{c}\text { Art. De relevância para } \\
\text { construção do artigo }\end{array}$ & 0 & 1 & 0 & 0 & 1 \\
\hline Total & 12 & 1 & 474 & 27 & 514 \\
\hline
\end{tabular}

Fonte. Dados da pesquisa, 2020.

Foi encontrado apenas estudos em inglês, visto que nos países de língua portuguesa este tipo de pesquisa ainda não é bem desenvolvido, comparada com outros países. Com a análise dos títulos foram incluídas 50 pesquisas, e já com a leitura dos resumos foram excluídos 25 estudos, totalizando 25 artigos para construção do presente estudo.

Após a análise dos estudos, foi detectado uma grande variedade das temáticas abordadas, entretanto o que se mostrou em menor quantidade (3) foram manuscritos com a temática de $\mathrm{FN}$ e doenças erradicadas (DE). Além disso, foi observado também que os artigos abordavam nos seus textos uma boa quantidade de informações sobre vacinação e $\mathrm{DE}$, mostrando a existência de uma ampla gama de estudos (8) nessa vertente (gráfico 1). 
Gráfico 1 - Temáticas presentes nos artigos.

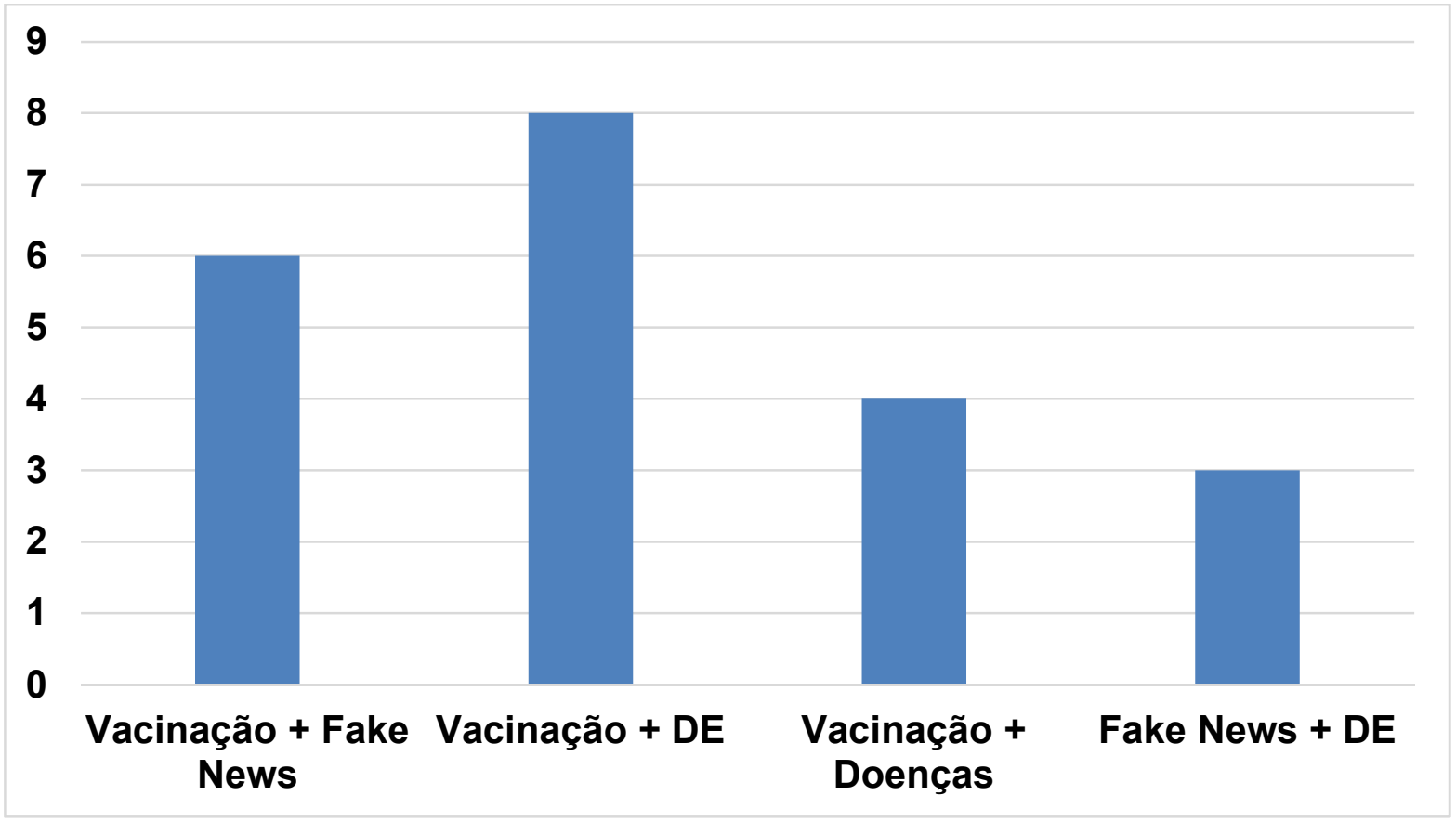

Fonte: Dados da pesquisa, 2020.

No gráfico 1, as evidências mostram, consistentemente, que as vacinas são seguras, eficazes e econômicas, diminuindo o custo com a saúde, visto que pessoas imunizadas ficam menos doentes. Entretanto, a mídia social tem sido a principal plataforma para desinformações, em diversos âmbitos da saúde, até mesmo entre pessoas da área. A informação, por vezes, pode chegar duvidosa e causar certa indagação perante os profissionais, sendo também, em alguns casos, vítimas desse processo (DANIELSON; MARCUS; BOYLE, 2019).

As notícias falsas que mais são repassadas sobre a vacinação, são: o organismo receberá uma carga do vírus que causa a doença e que o indivíduo vai adoecer. Entretanto, Nuismer et al. (2016) mostra que as vacinas são fracamente transmissíveis e que possuem uma alta capacidade de erradicar doenças, quando há políticas públicas, bem como aceitação da população perante a vacinação.

Durante surtos de doenças infecciosas emergentes, é comum recorrer às mídias sociais para buscar informações, bem como obter cuidados de como se proteger das doenças, dentre outras coisas. Tendo em vista isso, é de fundamental importância uma maior teorização das mídias digitais, bem como ferramentas nas 
redes sociais para que diminuam mais o compartilhamento de $\mathrm{FN}$, além da necessidade de rigor metodológico maior, para que o público fique mais embasado da verdade do que mentira (TANG; BIE; PARK; ZHI, 2018).

É importante atrelar a função das redes sociais durante surtos de doenças, desempenhando um papel de alertar a população, quanto a fonte, segura e veracidade. Além disso, o sentimento que a mídia social aflora na população, é raiva e medo, perante aos acontecimentos do momento. Também, uso da mídia social pode aumentar significativamente a prevenção, bem como o comportamento através das duas emoções auto-relevantes e da percepção de risco do público, podendo monitorar a aceitação das vacinas por essa via de comunicação social (OH; LEE; HAN, 2020).

Há um entendimento limitado do porquê certos indivíduos, sociedades e instituições são mais vulneráveis a notícias falsas sobre saúde. Talvez isso seja vantajoso, pois a promoção da saúde e pesquisadores de saúde pública agora prestam atenção considerável ao potencial das mídias sociais como ferramenta para difundir informações relacionadas à saúde, empregando telefones inteligentes e outras tecnologias móveis em intervenções preventivas. Embora a internet ofereça imensas oportunidades, também reduz o custo de geração e disseminação de informações, permitindo desinformação e histórias sensacionalizadas para propagar (WANG et al., 2019).

Kang et al. (2017), observam o sentimento atual das vacinas nas mídias sociais, por meio da análise de informações das redes de disseminação em sites compartilhados no Twitter nos Estados Unidos, vistos de forma negativa, diminuindo a cobertura vacinal, aumentando o risco de surtos de doenças. A prevalência do sentimento negativo de vacina foi demonstrada através de diversas mensagens, enquadrado em torno do ceticismo e desconfiança das organizações governamentais que comunicam evidências científicas apoiando benefícios da vacina. Dessa forma, estudo sintetiza evidência quantitativa e qualitativa de uma abordagem interdisciplinar para entender melhor fatores complexos da hesitação da vacina na comunicação em saúde pública, para melhorar a confiança da vacina e cobertura vacinal nos Estados Unidos. 
Após décadas de redução nas doenças evitáveis por vacina em todo o mundo, houve um reaparecimento de enfermidades na infância que já foram erradicadas. $O$ ressurgimento de afecções como poliomielite é consequência de eventos multifatoriais, como a falta de recursos em países pobres e devastados pela guerra, que levam à diminuição da vacinação (DREISINGER; LIM, 2019).

De acordo com Liria (2017), a diminuição das taxas de vacinação, por sua vez, tem duas origens: sequência de problemas de suprimento devido a desordens sociais e guerra em alguns países ou causado por uma inconcebível decisão voluntária de não se vacinar ou de não vacinar as crianças.

De acordo com Teoh (2019), a mídia social possui a capacidade de combinar a credibilidade da persuasão interpessoal com os meios de comunicação em massa, resultando em uma atitude ou comportamento desejado entre um grande grupo de pessoas. E no âmbito da saúde não seria diferente, os meios de comunicação podem ser eficazes na informação à população, entretanto podem haver algumas informações incorretas sobre o que se repassa, sendo necessário observar a sua veracidade.

Liria (2017) e Glanz et al. (2017) retratam como a mídia social pode interferir positivamente na vacinação nas crianças, através de um estudo com mulheres grávidas, onde elas recebiam nas suas redes sociais, de forma anônima, publicidades falando positivamente das vacinas. Dessa forma, tal experimento mostrou que a interferência da mídia na web, de forma positiva, mostrou que as mulheres vacinariam seus filhos ao nascerem.

As notícias falsas podem prejudicar bastante uma população, como por exemplo na vacinação, como é o caso exposto por lacobucci (2019), que no Reino Unido a aceitação da população a vacinação vem diminuindo, causando ressurgimento de doenças já erradicadas, bem como o agravo de doenças que ainda estão em combate. É pertinente a comparação entre este fato e o que ocorreu na região de São Paulo, onde começou um surto de sarampo, no mês de outubro de 2019, na população, doença que já tinha sido erradicada no Brasil.

Jervelund (2018) retrata como as redes sociais estão transformando a disseminação do conhecimento e como isso implica para nossas percepções sobre vacinação. O autor relata que na Dinamarca houve um aumento epidemiológico do 
papilomavírus humano (HPV). Isso corrobora os resultados de lacobucci (2019), pois mostra o desafio atual e real de se controlar doenças com a vacinação, pela disseminação de informações falsas sobre as vacinas aplicadas na população.

Apesar das inúmeras políticas públicas presentes na Europa, como campanhas de vacinação, subsídio governamental e pesquisas de novas vacinas, a população está desacreditada da eficácia dessa alternativa de prevenção das enfermidades e de seu papel fundamental na saúde e no bem-estar social, o que acarreta no aumento das doenças que as vacinas tentam prevenir ou mesmo erradicar, causando risco de epidemias (BECHINI, 2019).

No estudo de Rabinowitz et al. (2016), o relato é sobre a possibilidade de ideologias contra a vacinação nos Estados Unidos, mostrando que pais de crianças recém-nascidas estão com medo de vacinar seus bebês, perante tanta campanha ideológica dos grupos anti-vacinação. O que se comprova com a pesquisa de Bozzola et al. (2018), pois possui dados falando sobre campanhas nas redes sociais dos grupos contrários à vacina, e como essas campanhas estão aumentando o número de pessoas sem vacinação nas populações.

Em dois períodos de epidemias no Brasil, a de febre amarela e a do zika vírus (mais recente), a desinformação e distribuição de notícias falsas foram enormes, bem como as postagens em mídias sociais, que constroem o vírus zika como não perigoso, enquanto demonstram desconfiança em pesquisa e prevenção levando a uma combinação "perfeita", na qual as medidas de controle das políticas públicas não estavam sendo tão eficazes, ao ponto de uma parcela da população não se vacinar contra a febre amarela e minimizar os cuidados com o zika vírus (HENRIQUES, 2018; SIMPSON; LO, 2019). 
Gráfico 2 - Doses aplicadas por ano.

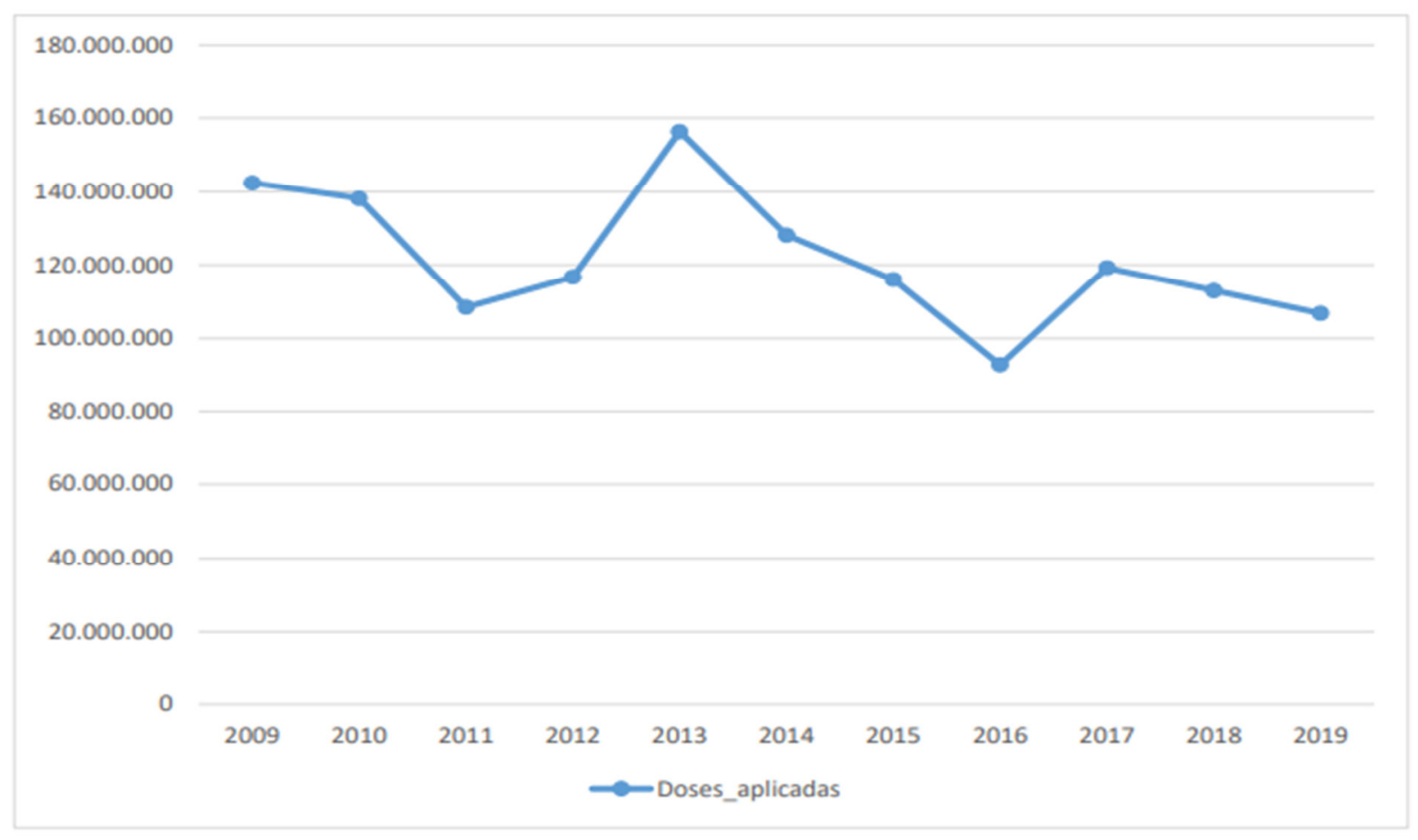

Fonte: Dados da pesquisa, 2020.

Podemos relacionar os movimentos anti-vacinas, bem como as FN propagadas, diminuindo $\mathrm{O}$ número de pessoas imunizadas. $\mathrm{O}$ auge das disseminações das informações falsas sobre vacinação e os crescentes grupos apoiadores da não vacinação, foi entre 2013 e 2014, na qual as pessoas começaram a duvidar da eficácia das vacinações e de sua veracidade, mostrando uma queda brusca no número de imunizações em todo o território nacional, onde o maior número de vacinações no Brasil foi em 2013, entretanto o menor em 2016. Gráfico 2.

Existe uma preocupação sobre o crescente sentimento anti-vacinal nos Estados Unidos e em outras partes do mundo. Com isso, grupos defensores das vacinas, desenvolveram um programa no país, enominado $A$ Voice for Choice, que corresponde a uma página na internet com o intuito de mostrar notícias verídicas sobre vacinação, bem como artigos científicos apresentando a verdadeira informação a quem visita o site. Dessa forma, tal estudo teve um saldo positivo, visto que houve um alcance grande de pessoas e aumento significativo da vacinação na 
população local, sendo visto como uma solução viável de acabar, ou pelo menos amenizar, a propagação das notícias falsas (EVRONY; CAPLAN, 2017).

Os estudos de Tavoschi et al. (2020) e Evrony e Caplan (2017), demonstram - Twitter como uma ferramenta para auxiliar o monitoramento da opinião pública sobre vacinação no período de um ano, na qual usou a rede social como uma espécie de sentinela potencialmente útil para a avaliação da opinião pública a respeito das vacinas. Dessa forma, podendo, no futuro, contribuir efetivamente para o desenvolvimento de estratégias de comunicação e informação, bem como no âmbito da saúde.

De acordo com lacobucci (2019), no Reino Unido possuem o NHS England Information Standard, órgão que é responsável por informar a população da veracidade das informações, o que se assemelha com o portal presente no site do Ministério da Saúde aqui no Brasil, o qual recebem informações duvidosas e esclarecem no site para a população saber se a informação repassada é falsa ou não.

A correlação entre o comportamento nas redes sociais de disseminação de notícias falsas sobre vacinação, vinculada com um sentimento contra as vacinas e a saúde pública, serve de importante parâmetro para monitorar a taxa de vacinação com a disseminação das FN. Como por exemplo, a transmissão de doenças mostra que, se grupos de sentimentos contrários à vacina levam a grupos de indivíduos desprotegidos, a probabilidade de surtos de doenças aumenta consideravelmente. Sendo assim, as mídias sociais online podem fornecer acesso sem precedentes aos dados, permitindo ferramentas baratas e eficientes para identificar áreas-alvo para os esforços de intervenção governamental, de incentivo a vacinação (SALATHÉ; KHANDELWAL, 2011).

Organizações incentivadoras da vacinação desenvolveram algumas estratégias de resposta, incluindo a comunicação com abertura de maneira informada por evidências, criação de espaços seguros para motivar o diálogo do público, fomentar parcerias comunitárias e combater desinformação com cuidado (STEFFENS; DUNN; WILEY; LEASK, 2019).

Esclarece-se que a vacinação deu uma enorme contribuição para a saúde global. Duas grandes infecções, a varíola e a peste bovina, foram erradicadas. Além 
disso, a cobertura global de vacinação contra muitas doenças infecciosas importantes da infância aumentou significativamente desde a criação do Programa Expandido de Imunização da OMS em 1974 e da Aliança Global para Vacinação e Imunização em (GREENWOOD, 2014).

Greenwood (2014) mostra também o futuro das vacinações, como vacinação contra ebola e HIV, dando ênfase ainda mais aos benefícios das vacinas para a sociedade, combatendo doenças que podem levar à morte, bem como a tentativa de suas erradicações.

A perspectiva para toda essa problemática não é nada positiva, porém com o uso de programas como o fato ou fake do ministério da saúde, para combater a propagação das $\mathrm{FN}$, disponibilizando um número de WhatsApp para envio de mensagens da população. Vale destacar que o canal não será um tira dúvidas dos usuários, mas um espaço exclusivo para receber informações virais, que serão apuradas pelas áreas técnicas e respondidas oficialmente se são verdade ou mentira. Diminuindo a desinformação da população, constituindo uma ótima ferramenta para aumentar a proporção de imunização da população.

\section{CONCLUSÃO}

As vacinas possuem sua eficácia, imunizando a população das enfermidades e erradicando doenças letais. Entretanto, com os avanços e modernização da disseminação das informações, pelas mídias digitais e sociais, o número de fake news sobre vacinação vem aumentando, fazendo com que haja o ressurgimento de doenças já erradicadas, bem como dificultando a contensão de doenças ainda presentes na sociedade.

Além disso, é notório e preocupante, o crescente aumento dos grupos antivacina, que espalham campanhas nas redes sociais, influenciam a população a não se vacinar, ficando expostos, a qualquer tipo de doença, prejudicando a sua própria saúde, bem como a do próximo. Assim, evidencia-se a importância da promoção de medidas esclarecedoras, especialmente via Organização Mundial da 
Saúde (OMS), com o intuito de minimizar a alienação da população sobre esta temática tão importante para prevenção de doenças na sociedade.

\section{REFERÊNCIAS BIBLIOGRÁFICAS}

BECHINI, A; BOCCALINI, S; NINCI, A; ZANOBINI, P; SARTOR, G; BONACCORSI, G; GRAZZINI, M; BONANNI, P. Childhood vaccination coverage in Europe: impact of different public health policies. Journal homepage, v. 18, n. 7, p. 693-701, 2019.

BOZZOLA, E; SPINA, G; RUSSO, R; BOZZOLA, M; CORSELLO, G; VILLANI, A. Mandatory vaccinations in European countries, undocumented information, false news and the impact on vaccination uptake: the position of the Italian pediatric society. Italian journal of pediatrics, $v$. 1, n. 10, p. 1-4, 2018.

DANIELSON, L.; MARCUS, B.; BOYLE, L. Countering Vaccine Misinformation. AJN, v. 119, n. 10, p. 50-55, 2019.

DREISINGER, N; LIM, CA. Resurgence of Vaccine-Preventable Disease. Ethics in the Pediatric Emergency Department, v. 35, n. 9, p. 651-653, 2019.

EVRONY, A; CAPLAN, A. The overlooked dangers of anti-vaccination groups' social media presence. Human Vaccines \& Immunotherapeutics, v. 35, n. 6, 2017.

GLANZ, J.M; WAGNER, N.M; NARWANEY, K. J; KRAUS, C. R; SHOUP, J. A; XU, S; LEARY, K. J; OMER, S. B; GLEASON, K. S; DALEY, M. F. Web-based Social Media Intervention to Increase Vaccine Acceptance: A Randomized Controlled Trial. Pediatrics, v. 140, n. 6, p. 1-11, 2017.

GREENWOOD, B. and future The contribution of vaccination to global health: past, present. Phil. Trans. R. Soc., v. 21, n. 5, p. 1-10, 2014.

HENRIQUES, CMP. Two epidemics: the yellow fever and a lack of information. Rev Eletron Comun Inf Inov Saúde, v. 12, n. 1, p. 9-13, 2018.

IACOBUCCI, G. Vaccination: "fake news" on social media may be harming UK uptake, report warns. The bmj, v. 10, n. 11, p. 364-365, 2019.

JERVELUND, SS. How social media is transforming the spreading of knowledge: Implications for our perceptions concerning vaccinations and migrant health. SJPH, v. 1, n. 46, p. 167-169, 2018.

KANG, G. J; EWING-NELSON, S. R; MACKEY, L; SCHLITT, J. T; MARATHE, A; ABBAS, K. M; SWARUP, S. Semantic network analysis of vaccine sentiment in online social media. Vaccine, v. 52, n. 5, p. $1-18,2017$.

LIRIA, CRG. Resurgence of almost eradicated paediatric diseases and the importance of childhood vaccination. Enferm Infecc Microbiol Clin, v. 35, n. 9, p. 547-549,2017.

LOPES, A.; FRANCOLLI, L. Revisão sistemática de literatura e metassíntese qualitativa: considerações sobre sua aplicação na pesquisa em enfermagem. Texto contexto enfermagem, v. 17, n. 4, p. 771-8, 2008. 
MCCLURE, C.C; CARTALDI, J.R; O'LEARY, S.T. Vaccine Hesitancy:WhereWeAreand Where WeAreGoing. Clinical Therapeutics, v. 1, n. 1, p. 1-13, 2017.

NUISMER, SL; ALTHOUSE, BM; MAY, R; BULL, JJ; STROMBERG, SP; ANTIA, R. Eradicating infectious disease using weakly transmissible vacines. TRS, v. 34, n. 8, p. 1-7, 2016.

OH, S.H; LEE, S; HAN, C. The Effects of Social Media Use on Preventive Behaviors during Infectious Disease Outbreaks: The Mediating Role of Self-relevant Emotions and Public Risk Perception. Health Communication, 2020.

RABINOWITZ, M; LATELLA; L; STERN, C; JOST, J. Beliefs about Childhood Vaccination in the United States: Political Ideology, False Consensus, and the Illusion of Uniqueness. Plosone, v. 11, n. 7, p. 1-19, 2019.

SALATHÉ, M.; KHANDELWAL, S. Assessing Vaccination Sentiments with Online Social Media: Implications for Infectious Disease Dynamics and Control. Computational Biology, v. 7, n. 10, p. 1-8, 2011.

SIMPSON, A. L.; LO, C. C. Risk society online: Zika virus, social media and distrust in the Centers for Disease Control and Prevention. Sociology of Health \& IIIness, p. 1-19, 2019.

STEFFENS, M. S.; DUNN, A. G.; WILEY, K. E.; LEASK, J. How organisations promoting vaccination respond to misinformation on social media: a qualitative investigation. BMC Public Health, v. 30, n. 19, p. 1-12, 2019.

TANG, L.; BIE, B.; PARK, S. F.; ZHI, D. Social media and outbreaks of emerging infectious diseases: A systematic review of literature. American Journal of Infection Control, 2018.

TAVOSCHI, L; QUATTRONE, F; D'ANDREA, E; DUCANGE, P; VABANESI, M; MARCELLONI, F; LOPALCO, P.L. Twitter as a sentinel tool to monitor public opinion on vaccination: an opinion mining analysis from September 2016 to August 2017 in Italy. Human Vaccines \& Immunotherapeutics, 2020.

TEOH, D. The Power of Social Media for HPV Vaccination-Not Fake News. ASCO EDUCATIONAL BOOK, v. 10, n. 12, p. 75-78, 2019.

WANG, Y; MCKEE, M; TORBICA, A; STUCKLER, D. Systematic literature review on the spread of health-related misinformation on social media. Social Science \& Medicine, v. 2, p. 1-49, 2019.

WASZAK, P.M; KASPRZYCKA-WASZAK, W; KUBANEK, A. The spread of medical fake news in social media $\boxminus$ the pilot quantitative study. Health Policy and Technology, v. 18, p. 1-17, 2018. 